\title{
CHARGED-PARTICLE EMISSIONS FROM DEUTERIDED METALS
}

\author{
F. W. KEENEY
}

Particle Physics Research Co., LLC, 2000 Linda Flora Dr., Los Angeles, CA 9007 ry

S. E. JONES

Department of Physics and Astronomy, Brigham Young University, Provo, UT 84604

\section{A. C. JOHNSON}

Particle Physics Research Co., LLC, 2000 Linda Flora Dr., Los Angeles, CA 9007r

\section{P. L. HAGELSTEIN}

Dept.of Electrical Engineering,, Massachusetts Inst. of Technology, Cambridge, MA 02139

G. HUBLER

U.S Naval Research Laboratory, Code 6370 Washington, D.C. 20375

D. B. BUEHLER

Particle Physics Research Co., LLC, 2000 Linda Flora Dr., Los Angeles, CA 90077

\author{
F. E. CECIL
}

Department of Physics, Colorado School of Mines, Golden, CO 80401

M. R. SCOTT AND J. E ELLSWORTH

Department of Physics and Astronomy, Brigham Young University, Provo, UT 84604

\begin{abstract}
We present evidence for energetic charged particles emanating from partiallydeuterided titanium foils $\left(\mathrm{TiD}_{x}\right)$ subjected to non-equilibrium conditions. To scrutinize emerging evidence for low-temperature nuclear reactions, we investigated particle yields employing three independent types of highly-sensitive, segmented particle detectors over a six-year period. One experiment measuring neutron emission from $\mathrm{TiD}_{x}$ foils showed a background-subtracted yield of $57 \pm 13$ counts per hour. (The neutron experiments are discussed in a separate paper in this proceedings.) A second experiment, using a photo-multiplier tube with plastic and glass scintillators and $\mathrm{TiD}_{x}$ registered charged particle emissions at 2,171 \pm 93 counts/hour, over 400 times the background rate. Moreover, these particles were identified as protons having $2.6 \mathrm{MeV}$ after exiting the $\mathrm{TiD}_{x}$ foil array. In a third experiment, coincident charged particles consistent with protons and tritons were observed with high reproducibility in two energy-dispersive ion-implanted detectors located on either side of 25-micron thick Ti foils loaded with deuterium.
\end{abstract}




\section{Introduction}

The first innovations towards producing our repeatable charge particle fusion emissions were provided in 1997 by F. W. Keeney ${ }^{1,2}$ of Particle Physics Research Co. who required more advanced particle detectors and contracted S. E. Jones ${ }^{3}$ of Brigham Young University to conduct the experiments.

We note that since 1989 there have been a number of serious attempts to observe nuclear effects in metal deuterides. Particularly relevant to the present work are experiments of Cecil, ${ }^{4}$ Hubler, ${ }^{5}$ Menlove, ${ }^{6}$ Jones, ${ }^{7}$ and Wolf. ${ }^{8}$ In all these cases, lowlevel neutron or charged-particle emissions from metal deuterides were seen by experienced individuals working with excellent detectors. The results were not widely published, particle identification was problematic and irreproducibility remained a significant issue. Now, about a decade later, we wish to revisit this problem with fresh ideas and considerable new experimental evidence.

For the most part, neutron observations reported as early as $1989^{6,9}$ have not been widely accepted. Screening at close range between deuterons in $\mathrm{TiD}_{x}$ is expected to be similar to that found in the $\mathrm{D}_{2}$ molecule and insufficient to produce measurable d-d fusion as concluded by Koonin and Nauenberg. ${ }^{10}$ Most early attempts at prominent laboratories to replicate such experiments were generally not successful and the situation has also been clouded by the controversy surrounding widely-publicized claims of aneutronic excess heat as reported by Huizenga. ${ }^{11}$

Encouraged by our neutron results, ${ }^{1}$ and wishing to also measure chargedparticle emissions, we designed two additional detection systems. The first system incorporated a plastic scintillator mounted on a glass scintillator, mounted in turn on a photo-multiplier tube (PMT). We observed charged-particle emissions in excess of 2100 counts per hour. Moreover, using an aluminum energy-degrader in the middle of a high-yield sequence of runs, we were able to identify the charged particles being emitted as protons carrying $2.6 \mathrm{MeV}$ after exiting a $\mathrm{TiD}_{x}$ foil, as we shall demonstrate. The second system incorporated the use of two independent ion-implanted silicon detectors (I-IDs). Our goal was to search for coincident (simultaneous) proton and triton signals produced in opposing directions according to the fusion reaction $\mathrm{d}+\mathrm{d} \rightarrow \mathrm{p}(3.02 \mathrm{MeV})+\mathrm{t}(1.01 \mathrm{MeV})$. Repeatability for $>2$-sigma effects approached $80 \%$ with these low-background, high-sensitivity dualcoincidence experiments. To reduce background effects, both sets of experiments were conducted in an underground laboratory beneath the Provo campus. 


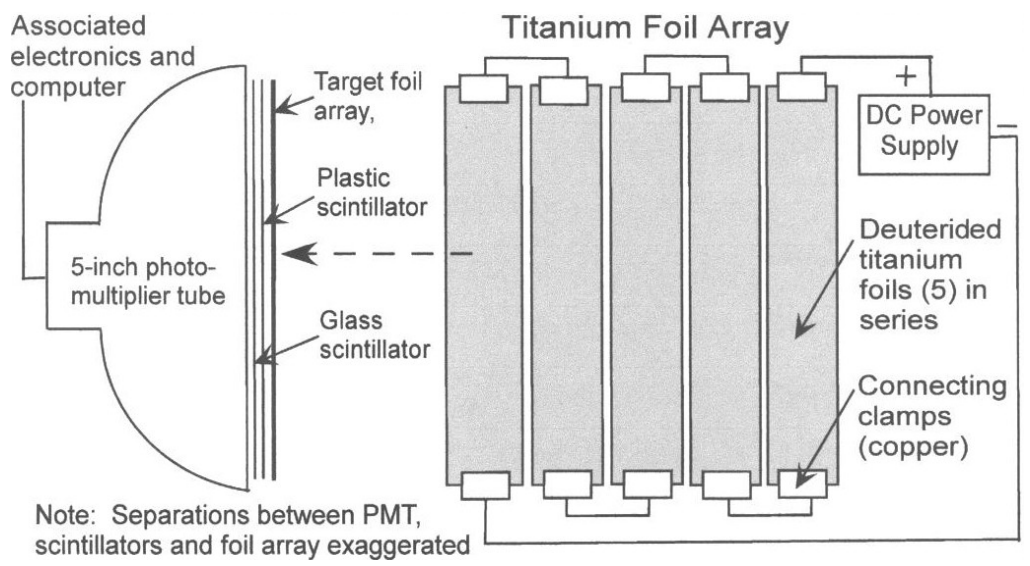

Figure 1. Foil array and charged-particle spectrometer system.

\section{Charged particle detection with a PMT/dual-scintillator system}

Our first charged-particle spectrometer (Fig. 1) incorporated a $0.0078 \mathrm{gm} / \mathrm{cm}^{2}(76$ $\mu \mathrm{m})$ thick plastic scintillator adhered onto a thicker glass scintillator $\left(0.375 \mathrm{gm} / \mathrm{cm}^{2}\right)$ which was glued onto the face of a 12.7 -cm diameter photo-multiplier tube (PMT). ${ }^{7}$ The detector was housed in a light-tight box equipped with electrical feed-throughs. PMT pulses were digitized at $100 \mathrm{MHz}$ over a $160 \mu$ s window. Pulse-shape analysis allowed us to distinguish narrow plastic pulses from glass pulses which are broader in time. When a particle passed through the plastic into the glass, the resulting combined pulse was broad and therefore interpreted as a glass pulse. The integrated area under the pulse reflects the light output of the scintillator(s) which corresponds to the energy of the incident charged particle. As shown in Fig. 2, the plastic scintillator has a non-linear response in that the light output depends on particle energy and identity. The composite spectrometer is also an effective cosmic-ray veto counter since nearly all cosmic rays entering the thin plastic scintillator must also pass through the glass scintillator, producing a characteristic broad (glass-like) pulse seen by the PMT. Cosmic ray pulses were therefore identified and efficiently eliminated. This dual-scintillator counter is a novel detector having the advantage of achieving large-area charged-particle detection conveniently. ${ }^{7}$

Titanium foils for these experiments were $20 \times 90 \mathrm{~mm}$ and 0.25 or $0.025 \mathrm{~mm}$ thick (both thicknesses were tried). The foils were placed inside a stainless-steel cylinder $35 \mathrm{~cm}$ in length by $2.5 \mathrm{~cm}$ outside diameter. The cylinder was evacuated to about $2 \times 10^{-5}$ Torr while being heated with Ti foils inside to approximately 400 ${ }^{0} \mathrm{C}$. At this temperature, the cylinder was pressurized with deuterium gas at $15 \mathrm{psi}$. After a 30 -second soak, the cylinder was re-evacuated, thus flushing out unwanted gasses from the cylinder. At approximately $500{ }^{\circ} \mathrm{C}$, the cylinder was pressurized 


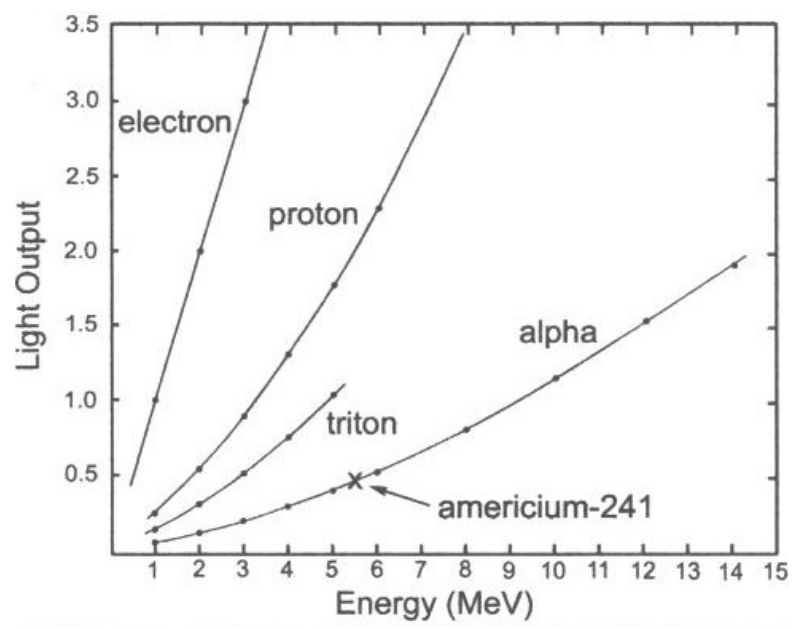

Figure 2. Light output for particles stopping plastic scintillator.

with deuterium gas at approximately $40 \mathrm{psi}$ and the inlet valve was closed. A drop in pressure (to about $10 \mathrm{psi}$ for the $0.25 \mathrm{~mm}$-thick foils) clearly demonstrated that the foils within the cylinder became deuterided. By measuring the mass of the foils before and after deuteriding, we found typical d-loadings of 0.5 to 1.4 deuterons per titanium atom. Typically five $\mathrm{TiD}_{x}$ foils were assembled in a picket-fence-style array as shown in Fig. 1, then placed on the surface of the detector. The associated electronics were activated and non-equilibrium conditions in the foils were produced by Joule heating.

We searched particularly for protons from the fusion of two deuterons according to the reaction

$$
\mathrm{d}+\mathrm{d} \rightarrow \mathrm{p}(3.02 \mathrm{MeV})+\mathrm{t}(1.01 \mathrm{MeV}) .
$$

The $3-\mathrm{MeV}$ proton is sufficiently penetrating to escape from about $50 \mu \mathrm{m}$ (or less) of titanium foil and produce a signal in the energy dispersive detector. On the other hand, exiting tritons have energies below the threshold for this detector. The PMT-detector has the virtue of a large surface area relative to ion-implanted silicon detectors (experiments described below), so that a relatively large sample can be studied in each experimental run.

Fig. 3 displays the response of the charged-particle spectrometer to $5.45-\mathrm{MeV}$ alphas particles from an americium-241 source. The pulse-area histogram reflects the $5.45-\mathrm{MeV}$ energy of the alphas and shows the resolution of $760 \mathrm{keV}$. These data along with Fig. 2 permit energy calibration of the spectrometer for plasticscintillator pulses, depending on incident particle type. Alphas of this energy all stop in the plastic scintillator and so produce characteristic narrow pulses.

Fig. 4 displays the spectrum obtained from a 52-minute background run. In an earlier study, we demonstrated that the majority of the background 


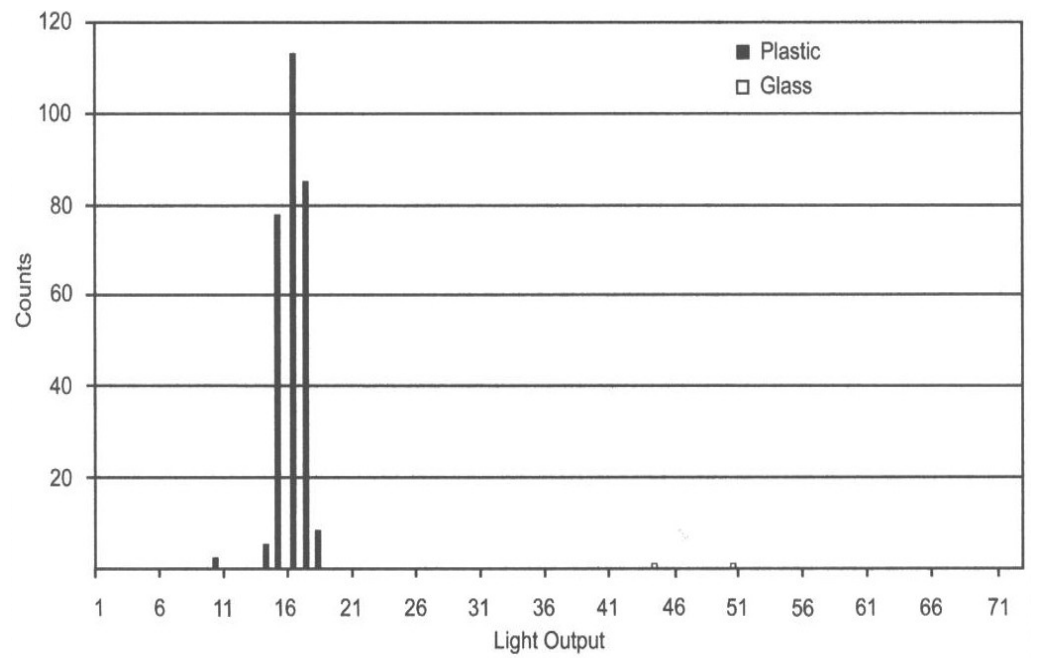

Figure 3. Light output from dual plastic/glass scintillator system for americium-241 source.

comes from minimum-ionizing cosmic rays and these produce predominately glass scintillations. ${ }^{7}$ The distribution of plastic-pulses is featureless over the background run as shown in Fig. 4. The glass-like pulses are seen to be more frequent but still at a very low rate. Radon was also of concern in these studies since it produces alpha energies up to $\sim 10 \mathrm{MeV}$ and is present in laboratory air. A prominent decay product, Bi-212, beta-decays to Po-212 which then decays via alpha emission with

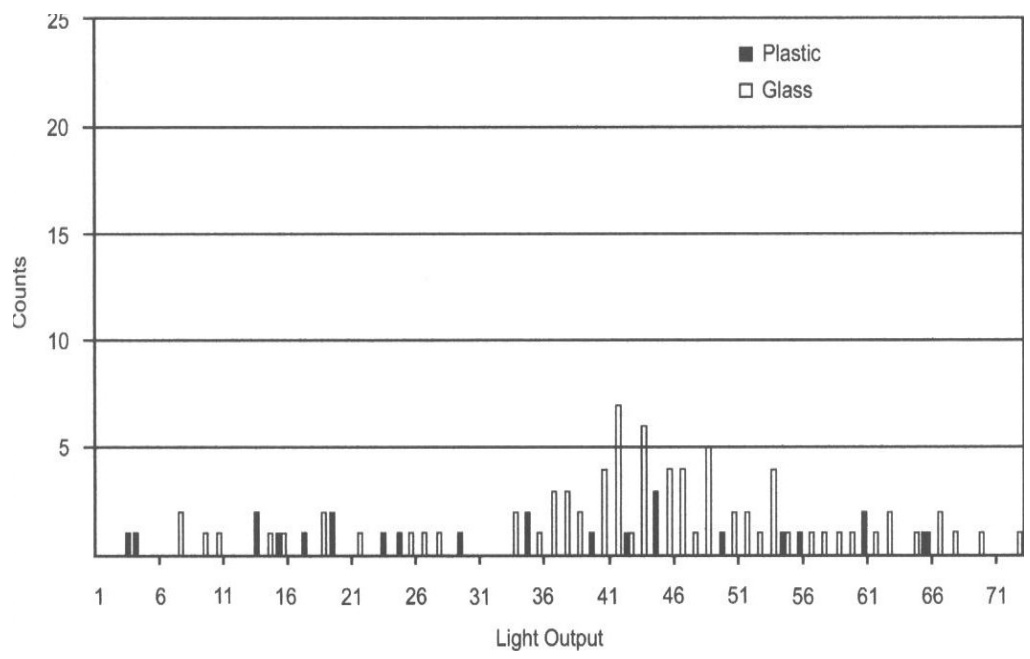

Figure 4. Spectrum obtained from a 52-minute background run, dual-scintillator detector. 


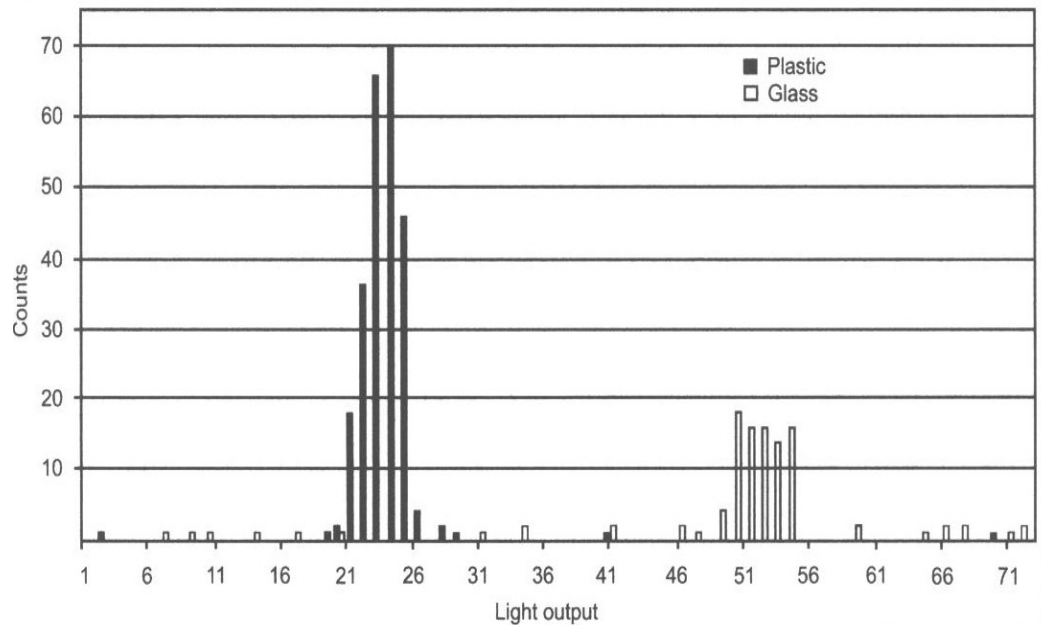

Figure 5. Spectrum from $\operatorname{TiD}_{x}$ array, 21 minutes with dual-scintillator detector.

a half-life of $0.3 \mu \mathrm{s}$. Fast digitization of pulses enabled us to clearly identify this decay sequence and eliminate such events, which proved to be few.

Fig. 5 shows results from a 21-minute run for a set of 3A-Joule heated, deuterium-loaded, titanium foils. We observed two clear groupings, corresponding to signals in the plastic scintillator and in the glass scintillator. Clearly, many particles registered only in the plastic scintillator while others penetrated through the plastic and produced dominating signals in the glass scintillator. Thus we observed two clear peaks completely different from anything ever observed during background runs. The charged particles penetrated through the plastic about onethird of the time to produce glass-dominated pulses, presumably at near-normal angles, while the remainder stopped in the plastic (see Fig. 6).

For the particles producing plastic-scintillator pulses, the peak in Fig 5 is approximately 1.4 times the light output of the americium alpha peak (Fig. 3). The light-output/energy calibration plot (Fig. 2) shows that these could be (a) alphas having $7.2 \mathrm{MeV}$, (b) tritons having $3.7 \mathrm{MeV}$, (c) deuterons having $3.1 \mathrm{MeV}$, (d) protons having $2.4 \mathrm{MeV}$, or (e) electrons with $0.7 \mathrm{MeV}$ (approximately; see Table 1). Protons with $2.4 \mathrm{MeV}$, with sufficiently off-normal angles to stop in the plastic scintillator, could arise from d-d fusion events originating about $12 \mu \mathrm{m}$ deep in the $\mathrm{TiD}_{x}$ foil, as diagramed in Fig. 6; see also Table 1.

To discriminate between particle types, a $19 \mu \mathrm{m}$-thick aluminum foil degrader was inserted between the $\mathrm{TiD}_{x}$ foils and the detector, with the results shown in Fig. 7. The plastic-pulse peak broadened significantly and the peak moved lower in energy, consistent with charged particles traversing a degrader (with small to large angles). Of all the particles mentioned, the implied energy loss is most consistent with protons (see Table 1), although the energy loss is somewhat smaller than 


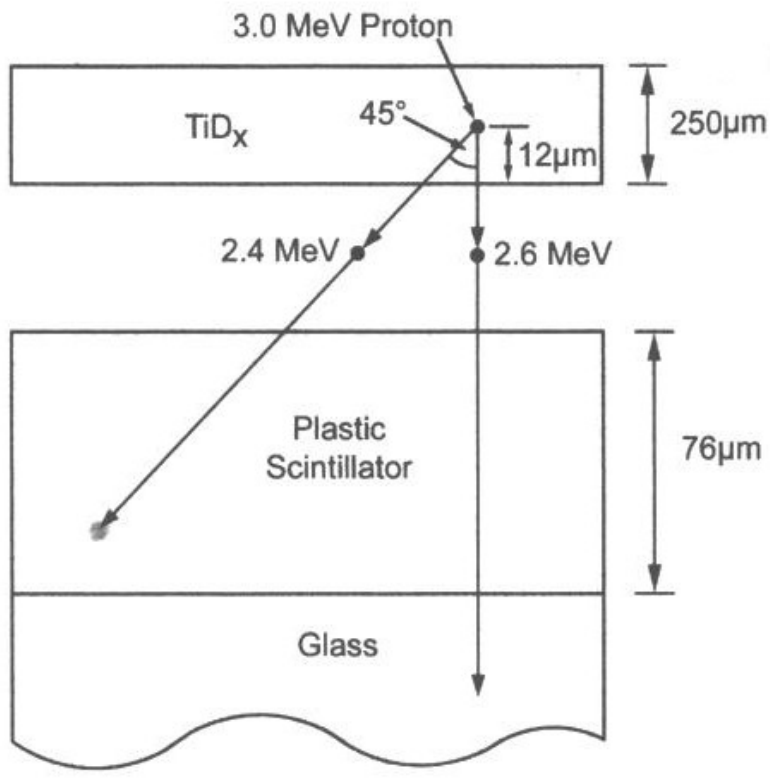

Figure 6. Schematic of plausible origin of $3 \mathrm{MeV}$ protons degrading through differing thicknesses of titanium at different angles in order to produce glass plus plastic pulses of observed energies.

might be expected, a result which may stem from the increased light-reflectivity of the aluminum foil degrader compared to the titanium-foil array. The glass-pulse

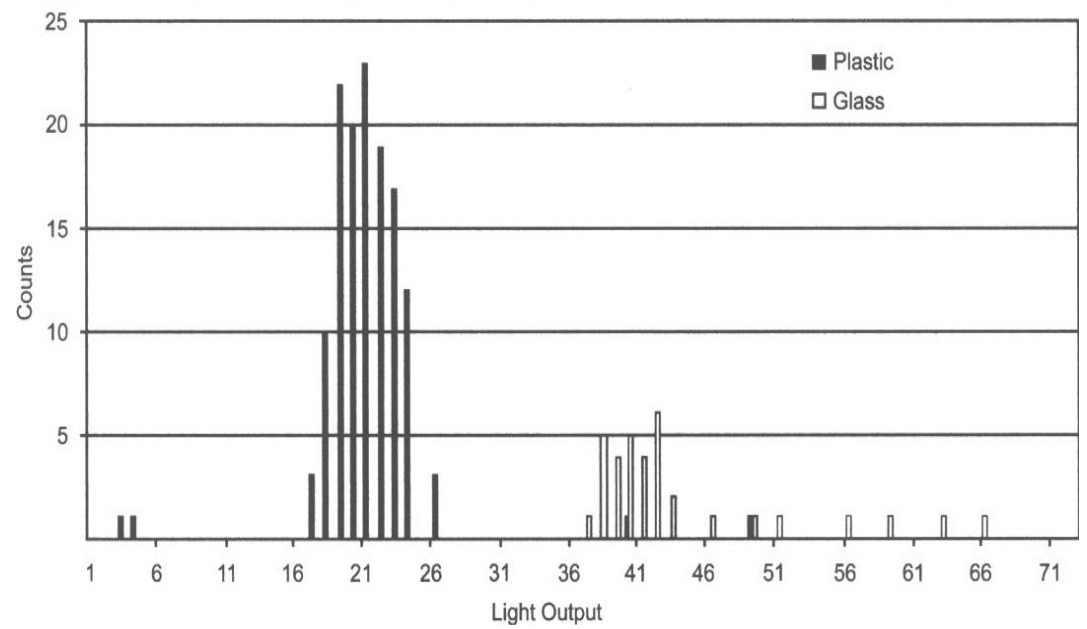

Figure 7. Spectrum from $\operatorname{TiD}_{x}$ array on top of 19 micron aluminum degraded for 5.5 minutes with dual-scintillator detectors. 
Table 1. Charged-particle identification matrix (Energies in $\mathrm{MeV}$ ).

\begin{tabular}{|c|c|c|c|c|c|c|}
\hline & $\alpha$ & $\mathrm{t}$ & $\mathrm{d}$ & $\mathrm{p}$ & & $\mathrm{e}$ \\
\hline $\begin{array}{l}\text { Energy needed to penetrate } 19 \mu \mathrm{m} \\
\mathrm{Al} \text { and } 76 \mu \mathrm{m} \text { plastic scintillator, } \\
\text { calculated using } \text { SRIM }^{12}\end{array}$ & 10.5 & 4.1 & 3.5 & 2.6 & & 15 \\
\hline $\begin{array}{l}\text { Energy from observed plastic- } \\
\text { scintillator peak, from } \\
\text { light-output/energy calibration }\end{array}$ & $7.2 \pm 0.2$ & $3.7 \pm 0.1$ & $3.1 \pm 0.2$ & $2.4 \pm 0.1$ & 0.7 & \pm 0.1 \\
\hline $\begin{array}{l}\Delta \mathrm{E} \text { through } 19 \mu \mathrm{m} \mathrm{Al} \text { degrader, } \\
\text { calculated using } \mathrm{SRIM}^{12}\end{array}$ & 2.8 & 0.8 & 0.7 & 0.5 & $\approx$ & 0.1 \\
\hline $\begin{array}{l}\Delta \mathrm{E} \text { through } 19 \mu \mathrm{m} \mathrm{Al} \text { degrader, } \\
\text { observed }\end{array}$ & $0.4 \pm 0.2$ & $0.3 \pm 0.2$ & $0.3 \pm 0.2$ & $0.3 \pm 0.2$ & $<$ & 0.1 \\
\hline
\end{tabular}

spectrum (Fig. 7) shows there remains a peak due to the glass scintillator, although now fewer charged particles get through both aluminum and plastic to produce a broad pulse in the glass scintillator.

We now have sufficient data to disentangle the identity of these particles. Table 1 displays the energy required for particles of various types to just penetrate the aluminum and plastic films (so as to produce a glass-scintillator pulse at normal incidence), as determined using the SRIM code. ${ }^{12}$ We also show the energy of the particles based on the light output/energy curves of Fig. 4. Alphas would need $10.5 \mathrm{MeV}$ to penetrate the aluminum and plastic degraders, but the test without Al-degrader limits the energy to $7.2 \mathrm{MeV}$. This contradiction eliminates alphas as candidate particles. Deuterons and tritons would require $4.1 \mathrm{MeV}$ and $3.5 \mathrm{MeV}$ respectively to traverse the aluminum and plastic degraders, but would then (like alphas) deposit more energy while stopping in the plastic scintillator than observed. Electrons of $0.15 \mathrm{MeV}$ will just traverse the plastic and aluminum degraders, but these cannot deposit $0.7 \mathrm{MeV}$ in the plastic scintillator so electrons are also eliminated. The 2.6 MeV required for normal transmission of protons through the degrader films is consistent with $2.4 \mathrm{MeV}$ deposited in the plastic scintillator (without $\mathrm{Al}$ degrader but allowing for energy losses in titanium; see Fig. 6). Finally, the delta-energy in the aluminum degrader is most consistent with protons (see Table 1). The results and SRIM calculations are thus consistent only with protons originating with $\sim 3-\mathrm{MeV}$ from a region roughly $12 \mu \mathrm{m}$ deep in a $\mathrm{TiD}_{x}$ foil due to the fusion reaction of Equation (1).

These data were also scrutinized for the presence of "bursts", defined as two or more detected signals within less than $21 \mu \mathrm{s} .{ }^{6}$ Burst events were clearly seen with the $\mathrm{TiD}_{x}$ foils; an example is provided in Fig. 8. The broad pulse is characteristic of a charged-particle stopping in the glass scintillator, whereas the later narrow pulse is typical of a charged-particle stopping in the plastic scintillator. Note that the 


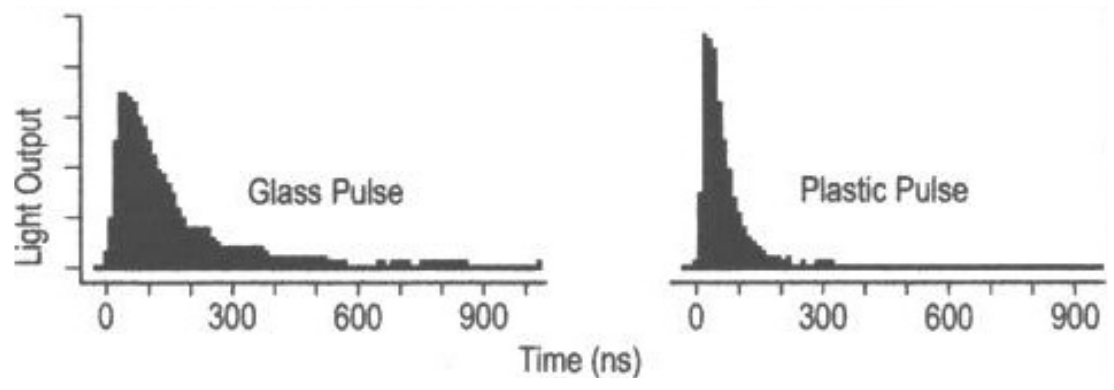

Figure 8. Burst event showing pulses from glass (left) and plastic scintillators.

total light output for a particle is determined from the integrated area under the pulse, so that the broader glass-scintillations will show much more light output than a plastic-pulse of the same pulse height. The plastic scintillations are calibrated for light output versus energy as explained above; the glass pulses are not calibrated but serve as indicators showing the particle penetrated the plastic scintillator into the glass. For the event in Fig. 8, the glass and plastic pulses were separated in time by $20.2 \mu \mathrm{s}$, as determined from the LeCroy waveform digitizer. For the $\mathrm{TiD}_{x}$ array discussed above, we found 27 events in 2490 seconds of data-acquisition having distinct glass and plastic (or plastic and plastic) pulses, separated in time by 1.08 to $20.2 \mu \mathrm{s}$ (the latter representing the maximum time window for burst events in this data set). There were zero such events in the 3104-second duration background run which immediately preceded these runs. (There were several glass-glass scintillation signals in both background and foreground runs, but these could readily arise from cosmic-ray showers.)

We conclude that low-multiplicity burst events do occur in $\operatorname{TiD}_{x}$ systems, in substantial agreement with earlier observations. ${ }^{1,5}$ Note that high-voltage breakdown in neutron-detectors' proportional-counter electronics a decade ago produced some spurious bursts of very high-multiplicity (30 or more putative neutrons in a burst event), ${ }^{3,6}$ but here we have verification of low-multiplicity bursts (which were never retracted) in an independent system without high-voltage breakdown. The physical meaning and significance of the charged-particle burst events remains to be determined.

Another striking feature of these data is the variation of count rates for single emissions with time, shown in Fig. 9. The background run had a rate of $3 \pm 2$ counts per hour in the light-output range of interest just before the $\mathrm{TiD}_{x}$ series began (compare Figs. 4 and 5). The initial Run $\mathrm{A}$ with $\mathrm{TiD}_{x}$ produced a yield of $887 \pm 87 \mathrm{cts} / \mathrm{h}$. The yield grew over the course of an hour to a striking $2,171 \pm$ $93 \mathrm{cts} / \mathrm{h}$ (Run E). This count rate is over 400 times the background rate and the statistical significance is over twenty standard deviations for this 907 -second run by itself. The rate was observed to decline after about 1.5 hours of running (Run F). These observations were followed by extensive tests of the equipment which proved 


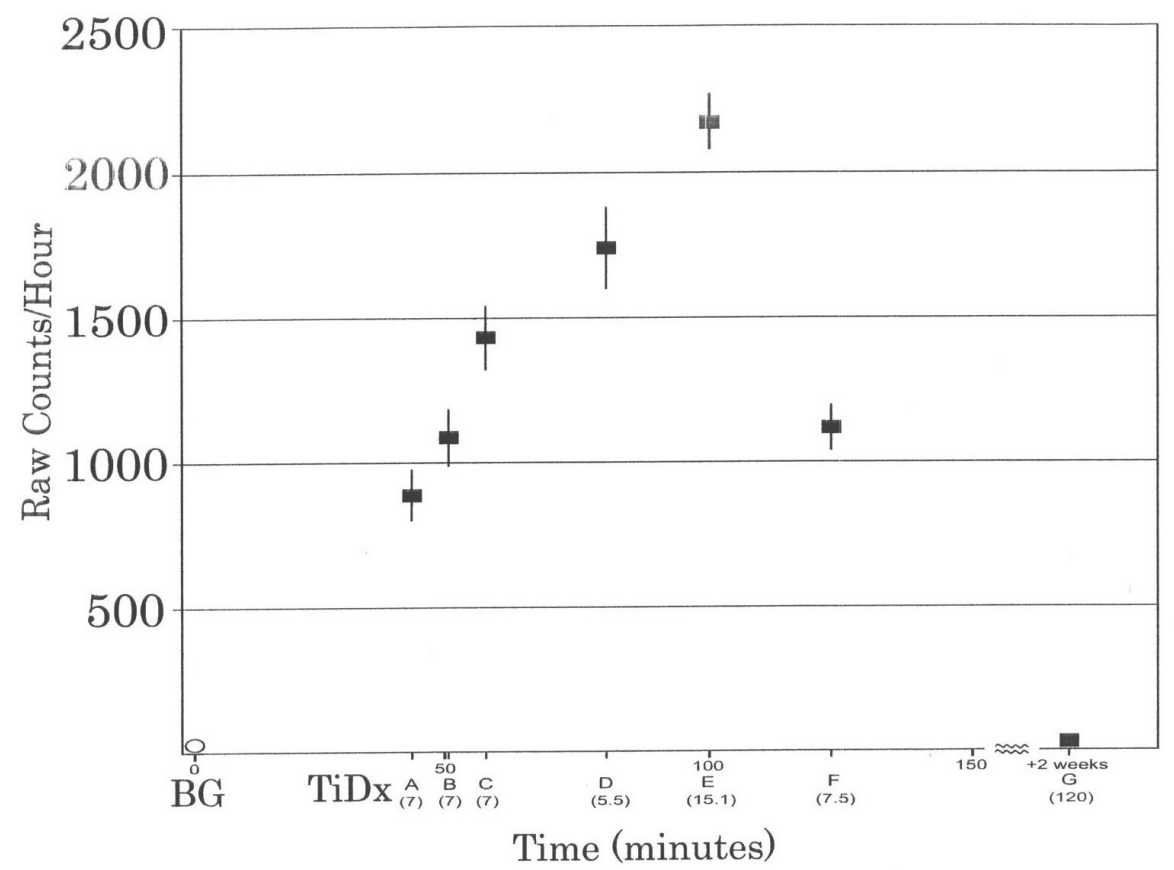

Figure 9. Charged-particle yields from a titanium deuteride array, plotted with run start-times relative to the end of the background (BG) run. The circle to the left near the bottom left corner is background. The run times for the different data points given are 7, 7, 7, 5.5, 15.1, 7.5, and 120 (all in minutes), going from left to right (A to $\mathrm{G}$ ). The rightmost data point was taken 2 weeks later.

to be functioning normally. Then the same $\mathrm{TiD}_{x}$ foils which had been so active were observed two weeks later, with the result of only $30 \pm 12 \mathrm{cts} / \mathrm{h}$ (Run G). These observations are consistent with detection of $3-\mathrm{MeV}$ protons produced by $\mathrm{d}-\mathrm{d}$ fusion occurring at a site approximately 12 microns deep in the partially-deuterided titanium, with fusion increasing in yield over time then decreasing dramatically. A prosaic (non-fusion) explanation for these data must fit this remarkable timedependence (Fig. 9), as well as the energies observed with and without aluminum degrader (Table 1 ). We next moved on to a very high-sensitivity dual-coincidence silicon-detector system which gave lower yields but considerably higher repeatability, described in the next section.

\section{Detection of coincident charged-particle emissions using ion-implanted silicon detectors}

Two $900 \mathrm{~mm}^{2}$ ion-implanted detectors (I-ID) were mounted in a small cylindrical vacuum chamber which also served as a Faraday cage for the detectors, refer to Fig. 10. Deuterided titanium foils 25 -microns thick were positioned between the two detectors. Our goal was to search for coincident (simultaneous) proton and triton signals produced in opposing directions according to the fusion reaction of Eqn. (1). 


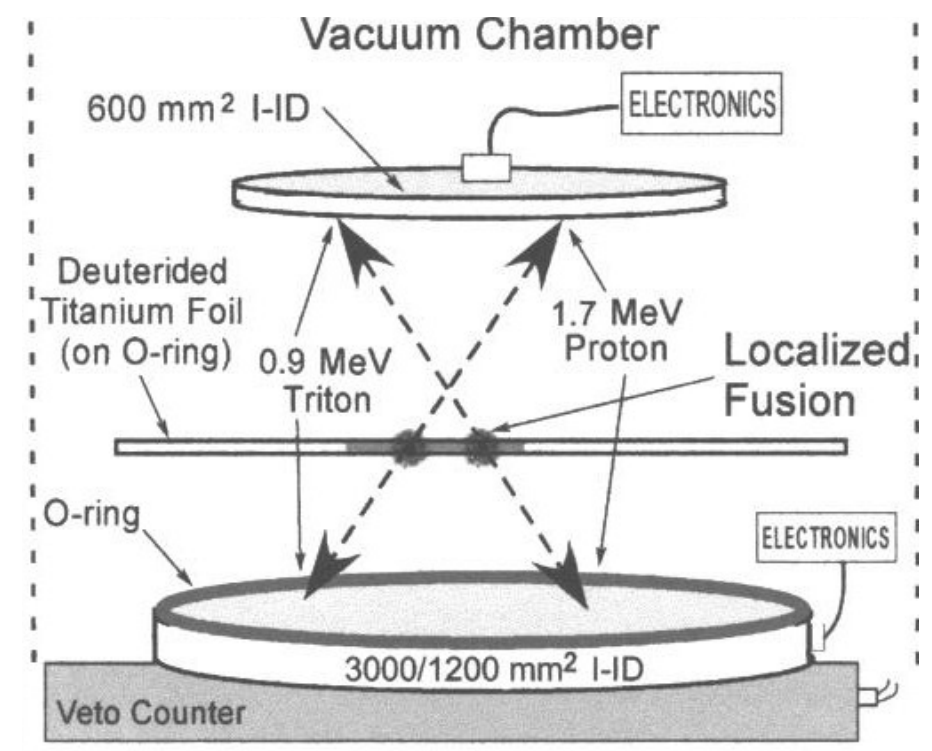

Figure 10. Coincident charged particle detector apparatus with two silicon (I-ID) detectors.

Because some nuclear particles must travel through some thickness of the foils to reach the detectors, the energies on arrival at the detectors are less than their originating energies. We performed Monte Carlo studies using the SRIM code to determine energies of arriving particles.

Typical experiments incorporated two titanium foils, 5 -cm in length, $0.025-\mathrm{mm}$ thick and $2.5 \mathrm{~cm}$ in width, processed as described in the previous section. We admitted enough $\mathrm{D}_{2}$ to bring the average $\mathrm{d} / \mathrm{Ti}$ ratio to approximately 1 to 1.6 for these experiments. The cylinder was then removed from the oven and allowed to cool slowly to room temperature for about one hour before we removed the foils. (Caution: the following procedure should be performed in a ventilated hood while using impermeable gloves and goggles.) Both surfaces of these foils were then further treated by application of lithium-deuteride powder then $\mathrm{D}_{2} 0+\mathrm{D}_{2} \mathrm{SO}_{4}(5: 1$ ratio) was cautiously added from a dropper. Vigorous reactions ensued, releasing deuterium gas (some of which presumably entered the foils) and providing localized heating in various degrees throughout the foils. The foils were rinsed to stop chemical reactions, then dried and mounted parallel to each other between the I-IDs. No Joule heating was employed during these particular experiments because of potential hazards to the detectors due to heating within the vacuum environment. Pulses from the two detectors were digitized using a dual-input LeCroy waveform digitizer over 160 microseconds and data stored using a PC/CAMAC system for again analyzing pulses off-line and differentiating noise (ragged shapes) from chargedparticles detected in the silicon detectors (broad, smoothly-decaying signals). The upper detector provided the trigger for the digitizer. We incorporated a $31 \mathrm{~cm}$ by 


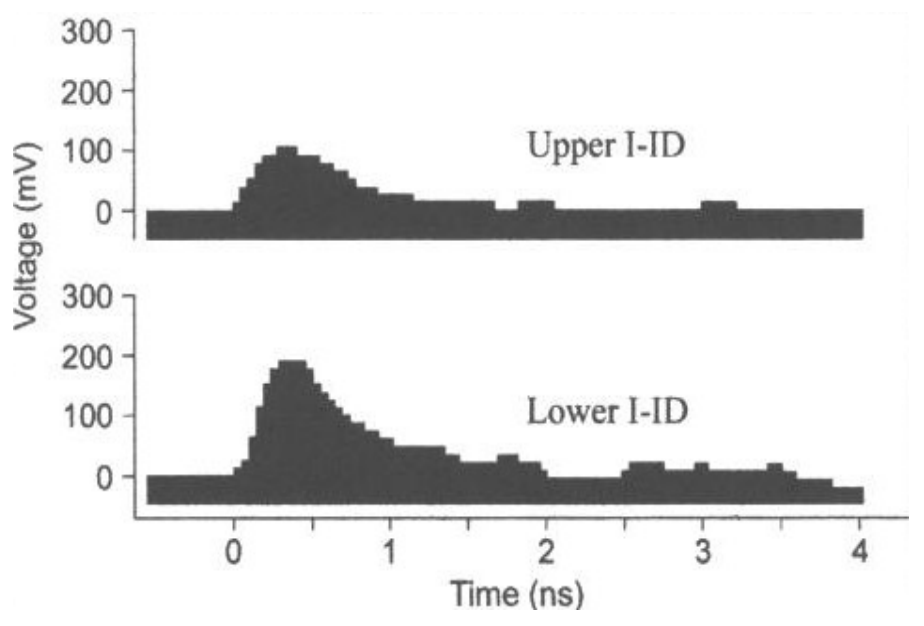

Figure 11. A typical dual-coincidence event.

$51 \mathrm{~cm}$ (plastic scintillator) cosmic-ray veto counter into the system, placed directly above the vacuum test chamber. A typical charged particle coincidence event is shown in Fig. 11.

The upper detector shows a pulse corresponding to approximately $0.9 \mathrm{MeV}$ while the lower detector shows a pulse corresponding to $1.7 \mathrm{MeV}$. Both I-IDs were calibrated using an Am-241 source in conjunction with a Canberra pulser. This event is consistent with a triton from $\mathrm{d}$-d fusion originating a few microns from the top surface of the $\operatorname{TiD}_{x}$ and traveling upwards while a proton passes through about 20 microns of titanium (again, losing energy) then strikes the lower detector [see the reaction of Equation (1)]. (The result would be plotted as a point at $(0.9,1.7)$ in Fig. 12.)

Fig. 12 shows data acquired for one $\operatorname{TiD}_{x}$ foil. Cosmic-ray vetoed events and non-coincident low-energy events are eliminated. We plot energy in the lower detector (y) versus energy in the upper detector (x) for coincident charged particles. We have a great deal of data acquired with this detector, and have worked to reduce background counts until now the background is only about 2.3 counts per day (Fig. 13). For this brief paper, we show data taken in July and August, 2003, acquired with one $\mathrm{TiD}_{x}$ foil and one $\mathrm{TiH}_{x}$ foil. The contrast of background data (Fig. 13, $\mathrm{LiH}$-treated $\mathrm{TiH}_{x}$ ) and foreground data (Fig. 12, LiD-treated $\mathrm{TiD}_{x}$ ) is clearly seen with many more coincident charged particles detected for the deuterided metal case. For the applied cuts (boxes) and excluding cosmic-ray vetoed events, we find just $(2.3 \pm 0.6)$ background counts per day, whereas the TiD foil produced $(9.5 \pm 1.0)$ foreground counts per day, giving a statistical significance of a nearly six standard deviations for these data.

In summary, there is indeed strong evidence for a significant effect consistent with coincident protons and tritons from $\mathrm{d}-\mathrm{d}$ fusion in metals. Other nuclear reactions are not excluded at this time, as we have not yet identified the charged 


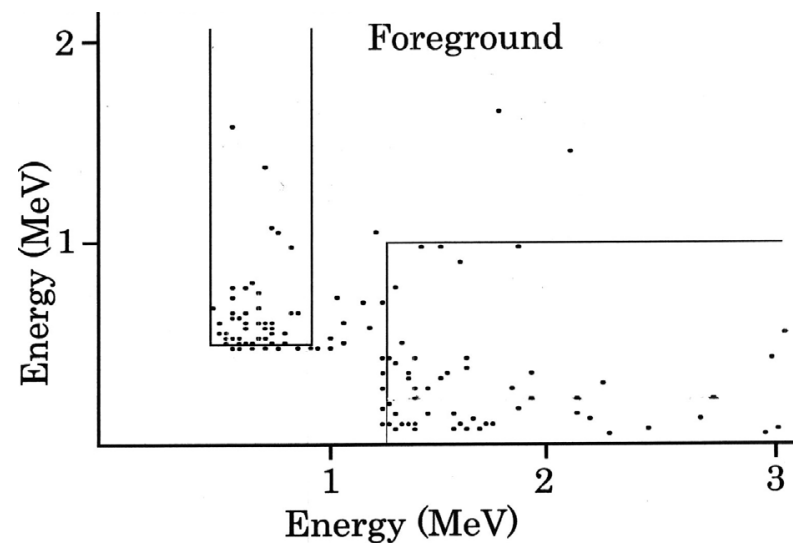

Figure 12. Data acquired for one TiDx foil using the BYU dual-coincidence silicon-detector spectrometer, 9.7 days. Energy in the lower detector (y) versus energy in the upper detector (x) is displayed for each event. Cosmic-ray vetoed events and non-coincident (below threshold) events are eliminated.

particles, although a delta-E/Energy telescope is contemplated. Effects due to natural radioisotopes such as radon as a possible source of artifacts were seriously investigated, but we could find no prosaic source that could produce back-to-back charged particles having the correlated energies observed, correlated with metal deuterides, especially considering the vacuum environment of the dual silicon-detector experiments.

\section{Conclusion}

Data presented in this paper provide strong evidence for a significant effect consistent with low level emission of protons and tritons from $\mathrm{d}-\mathrm{d}$ fusion in metals. The

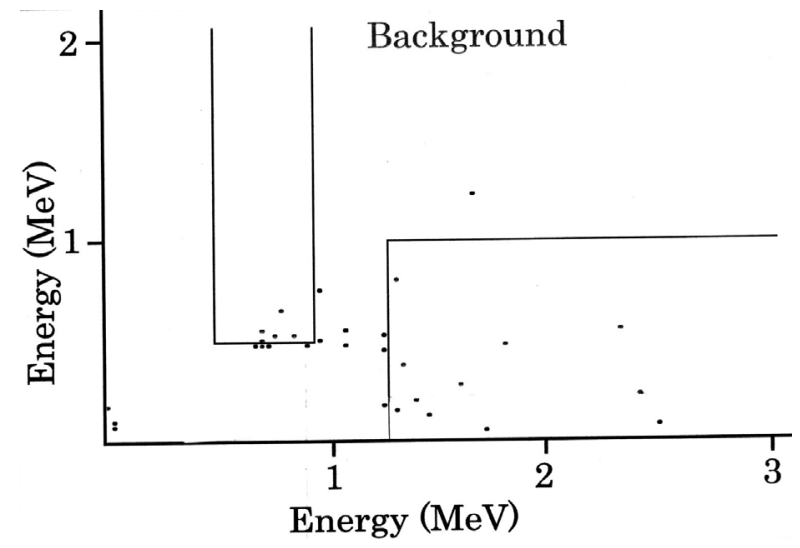

Figure 13. Background data acquired for a TiHx foil using the dual-coincidence spectrometer, 7.1 days. 
measured rates are $\sim 10^{-21}$ to $10^{-25}$ fusions/deuteron pair/s. Such an effect is seen within $20-80 \%$ of trials, using three different detection systems and several different methods of sample preparation. Observed yields varied from about 0.4 /hour to 2100 /hour and although active $\mathrm{TiD}_{x}$ volumes varied greatly, the reasons for the rate variations require further exploration. Based on what is known from solid state and nuclear physics, $\mathrm{d}$ - $\mathrm{d}$ fusion is not expected in $\mathrm{TiD}_{x} \cdot{ }^{10,11}$ Hence, we consider these experimental results to represent a new physical process of some kind. We have shown that special treatments are important to produce the effects; such as cleaning the foils, gas loading at high temperature, acid loading in the presence of $\mathrm{LiD}$, grooving and strong electrical fields in the foils. ${ }^{1,2}$ The relative importance of each treatment requires further study. There is a suggestion in the data that electrical current provides more consistent results and higher yields. ${ }^{1,2}$ It is possible that other nuclear reactions besides d-d fusion are involved. Also of great importance are detection systems designed for very low counting rates.

Among the advances represented by this work are significant nuclear particle yields at low temperatures, particle identifications, and high repeatability. These were achieved through innovative pre-processing and deuteriding of thin titanium foils and application of substantial electrical currents. ${ }^{1,2}$ It is speculated that microscopic variables in the foils, such as lattice/defect variations and surface conditions, prevented totally $100 \%$ reproducibility. Nevertheless, repeatability is sufficiently high that others should be able to reproduce these results.

\subsubsection{Acknowledgments}

The authors acknowledge support and useful input from Bart Czirr, Howard Menlove, Lawrence Rees, Lee Hansen, James Thorne, Robert Routsong, Joseph Young, Wes Lifferth; Seth, David and Nathan Jones, Dennis Cravens.

\section{References}

1. F. W. Keeney, S. E. Jones, A. C. Johnson, D. B. Buehler ,F. E. Cecil, G. Hubler, P. L. Hagelstein, et al, "Neutron Emissions from Deuterated Metal," (submitted to Physical Review C.)

2. F. W. Keeney, S. E. Jones, A. C. Johnson, U.S. Patent Application Serial No. 09/514,202, and International Patent Cooperation Treaty (PCT) Application No. PCT/US01/05344, "Low-temperature Nuclear Fusion under Non-equilibrium Conditions," Particle Physics Research Co., LLC, Los Angeles (2000).

3. S. E. Jones, "Chasing Anomalous Signals: The Cold Fusion Question," Acc. in Research, 8 55-58 (2000).

4. F. E. Cecil, H.Liu, D. Beddingfield, and C. S. Galovich, "Observation of Chargedparticle Bursts from Deuterium-Loaded Thin-Titanium Foil," American Institute of Physics: Conference Proceedings 228, NY (S.E Jones, F. Scaramuzzi, \& D. H. Worledge, eds.). See Chapter 2, "Charged-Particle Emissions," Anomalous Nuclear Effects in Deuterium/Solid Systems, p. 375 (1991).

5. G. P. Chambers, G. K. Hubler, and K. S. Grabowski, "Search for Energetic ChargedParticle-Reaction Products During Deuterium Charging of Metal Lattices," American Institute of Physics: Conference Proceedings 228, NY (S.E Jones, F. Scaramuzzi, \& D. 
H. Worledge, eds.). See Chapter 2, "Charged-Particle Emissions," Anomalous Nuclear Effects in Deuterium/Solid Systems, p. 383 (1991).

6. H.O. Menlove, and J.E. Swansen, Nuclear Technology 71, 497-505 (1985). H.O. Menlove, M.M. Fowler, E. Garcia, A. Mayer, M.C. Miller, R.R. Ryan and S.E. Jones, "Measurement of Neutron Emission from Ti and Pd in pressurized $\mathrm{D}_{2}$ Gas and $\mathrm{D}_{2} \mathrm{O}$ Electrolysis Cells," J. Fusion Energy 9, 495-506.

7. S. E Jones, T. K. Bartlett, D. B. Buehler, J. B. Czirr, G. L. Jensen, and J. C. Wang, "Preliminary Results from the BYU Charged-Particle Spectrometer," Chapter 2, "Charged-Particle Emissions," Anomalous Nuclear Effects in Deuterium/Solid Systems, p. 397

8. K.Wolf, EPRI reports now available at: http://lenr-canr.org/acrobat/PassellTOradiationd.pdf.

9. S. E. Jones, E. P. Palmer, J. B. Czirr, D. L. Decker, G. L. Jensen, J. M. Thorne, S. F. Taylor, J. Rafelski, "Observation of Cold Nuclear Fusion in Condensed Matter," Nature 338 737-740 (1989).

10. S. E. Koonin, and M. Nauenberg, "Cold Fusion in Isotopic Hydrogen Molecules," Nature 339, p. 690 (1989).

11. J. R. Huizenga, Cold Fusion: The Scientific Fiasco of the Century, University of Rochester Press, (1992).

12. J. F. Ziegler, Stopping and Range of Ions in Matter (SRIM-2000), http://www.srim.org/. 